\title{
Adsorption properties of modified eggshell on phosphorus in water
}

\author{
Xiuling $\mathrm{Li}^{1}$, Yaqing Liu ${ }^{1}$, Lanlan $\mathrm{Su}^{1}$, and Lei Xin*1 \\ ${ }^{1}$ College of Chemical and Biological Engineering, Hechi University, Yizhou GuangXi, 546300, China
}

\begin{abstract}
Modified eggshell adsorbent was prepared with 5\% potassium permanganate solution to adsorb phosphorus in simulated wastewater. Fourier transform infrared spectroscopy (FTIR), X-ray diffraction (XRD), and scanning electron microscopy (SEM) were used to clarify their phosphorus removal mechanisms. Isothermal and kinetic equations were fitted to explain the mechanism of phosphorus adsorption. The results showed that the optimum conditions were as follows: phosphorus concentration of 5 $\mathrm{mg} \cdot \mathrm{L}^{-1}$, wastewater $\mathrm{pH}$ of 2 , adsorbent dosage of $3.5 \mathrm{~g}$, adsorption time of $350 \mathrm{~min}$, phosphorus adsorption rate of modified eggshell was $90.81 \%$, and the experimental results were relatively stable. According to the characterization, the eggshell adsorbent has good crystallinity, irregular surface structure and abundant pore channels, and the main functional groups on the surface are hydroxyl $(-\mathrm{OH})$ and $\mathrm{C}-\mathrm{H}$ groups, which enhanced the adsorption effect.
\end{abstract}

\section{Introduction}

With the development of industrialization and the improvement of people's living standards, the problem of environmental pollution has become increasingly prominent. In recent years, the proportion of eutrophication in surface water in China has increased from $5 \%$ in the past to $55 \%^{[1]}$. One of the main elements leading to water eutrophication is phosphorus. If untreated phosphorous-containing wastewater is discharged into water, it will lead to a sharp decline in dissolved oxygen in water and a large number of aquatic organisms will die, which will affect the ecological balance of water ${ }^{[2]}$. Therefore, phosphorus removal from wastewater has become a hot topic in research. Due to its low cost and good effect, adsorption is a relatively common method for phosphorus removal ${ }^{[3]}$. The selection of adsorbent with low price and good effect has become a key factor in the treatment of phosphorouscontaining wastewater by adsorption. Some scholars at home and abroad have tried to use modified zeolite ${ }^{[4]}$, lanthanum-modified bentonite ${ }^{[5]}$, activated alumina ${ }^{[6]}$ and other adsorption and dephosphorization methods.

Eggs have high nutritional value and are the daily food for human consumption. With the increasing demand for eggs, a large number of eggshells are discarded as kitchen waste, which not only wastes resources but also pollutes the environment. Paying attention to the comprehensive utilization of discarded eggshells can provide a new way for its resource utilization $^{[7]}$. Although eggshell is a kind of solid waste, it is rich in calcium elements, it has a porous structure, a large specificsurface area and good gas and liquid phase adsorption performance.

In recent years, there have been many studies on the comprehensive utilization of discarded eggshells. Agarwal Animesh ${ }^{[8]}$, Mahtab Ahmad ${ }^{[9]}$, PARK Heung $\mathrm{Jai}^{[10]}$, I.A.Oke ${ }^{[11]}$ and so on all used discarded eggshells as adsorbents to adsorb heavy metals in water. N.Yeddou Mezenner ${ }^{[12]}$ made a kinetics and thermodynamic study of phosphate adsorption on iron hydroxide-eggshell waste. Rahmi ${ }^{[13]}$ made a study about the influence of heat treatment on eggshell particle as low cost adsorbent of methylene blue. It can be concluded that modified eggshell has a better adsorption effect and is a very good adsorbent.

\section{Material and Methods}

\subsection{Reagents and Instruments}

$\mathrm{KMnO}_{4}, \mathrm{NaOH}, \mathrm{KH}_{2} \mathrm{PO}_{4}, \mathrm{H}_{2} \mathrm{SO}_{4}, \mathrm{~K}(\mathrm{SbO}) \mathrm{C}_{4} \mathrm{H}_{4} \mathrm{O}_{6} \cdot 5 \mathrm{H}_{2} \mathrm{O}$, and $\mathrm{H}_{8} \mathrm{MoN}_{2} \mathrm{O}_{4}$ were purchased from Tianjin Guangfu Science and Technology Development Co. LTD. All reagents were of analytical grade and de-ionized water was used in all experiments. Simulated phosphoruscontaining wastewater was prepared by diluting a stock solution of $\mathrm{KH}_{2} \mathrm{PO}_{4}$.

The instruments were the following:

X-Ray Diffractometer (XRD); NICOLET 6700 Fourier infrared spectrometer (FTIR); Scanning Electron Microscope (SEM); Series $5100 \quad$ UV/visible spectrophotometers.

The treatment of phosphorous-containing wastewater by modified eggshell with potassium permanganate has

Corresponding author: 06063@hcnu.edu.cn 
not been reported. In this study, eggshell powder modified by $5 \%$ potassium permanganate solution was used as an adsorbent to explore the influence of different conditions on phosphorus adsorption performance. It not only provides new adsorption materials and theoretical support for phosphorus removal in wastewater, but also provides a new application way for eggshell treatment and disposal.

\subsection{Material pretreatment}

Collect eggshells from the canteen of our school and clean them with clean water, peel off the inner membrane of the shells, wash them with pure water for 2-3 times, put them into the oven for drying at $75{ }^{\circ} \mathrm{C}$, take them out and cool them down, then use a grinder to smash them and pass 80 mesh sieve for later use.

\subsection{Preparation of modified eggshell adsorbent}

About $300 \mathrm{~g}$ eggshell powder was weighed and modified by adding $5 \%$ potassium permanganate solution of a certain volume under stirring. It was put into a shaker at $25^{\circ} \mathrm{C}$ and shaken for 48 hours at $160 \mathrm{r} \cdot \mathrm{min}^{-1}$. Then it was centrifuged and washed with a large amount of pure water until $\mathrm{pH}$ neutral, dried and set aside.

\subsection{Calculation of phosphorus removal}

In this experiment, the removal rate and Adsorption quantitywere used as two evaluation indexes to study the phosphorus removal effect of the modified eggshell adsorbent.Phosphorus removal efficiency was calculated as follows:

$$
\eta=\left[\left(C_{0}-C_{t}\right)\right] / C_{t} * 100 \%
$$

where $\eta$ is the total phosphorus removal efficiency (\%), $\mathrm{c} 0$ is the initial total phosphorus concentration $\left(\mathrm{mg} \cdot \mathrm{L}^{-1}\right)$, and ct is the time-dependent total phosphorus concentration $\left(\mathrm{mg} \cdot \mathrm{L}^{-1}\right)$.

Adsorption quantity of Phosphorus was calculated as follows:

$$
Q_{t}=\left[\left(C_{0}-C_{t}\right) \times V\right] / m
$$

where Qt is adsorption quantity of Phosphorus $\left(\mathrm{mg} \cdot \mathrm{g}^{-1}\right), \mathrm{V}$ is the volume of waste water $(\mathrm{mL}), \mathrm{m}$ is dosage of adsorbent $(\mathrm{g})$.

\subsection{Single factor experiment of adsorption process}

\subsubsection{Influence of simulated wastewater initial concentration on total phosphorus removal rates}

The experimental conditions were fixed, that is, the $\mathrm{pH}$ value of each simulated wastewater sample was adjusted to 2 and the volume was $50 \mathrm{~mL}$, the fixed dosage of modified eggshell powder adsorbent was $4.0 \mathrm{~g}$, and the temperature of the shaker reactor was $25^{\circ} \mathrm{C}$, the speed was $160 \mathrm{r} \cdot \mathrm{min}^{-1}$, the adsorption time was $200 \mathrm{~min}$.

\subsubsection{Influence of adsorbent dosage on total phosphorus removal rate}

The experimental conditions were fixed, that is, the concentration of phosphorus-containing simulated wastewater was $10 \mathrm{mg} \cdot \mathrm{L}^{-1}, \mathrm{pH}$ value was adjusted to 2 and the volume was $50 \mathrm{~mL}$, the temperature of the shaker reactor was $25^{\circ} \mathrm{C}$, the speed was $160 \mathrm{r} \cdot \mathrm{min}^{-1}$, the adsorption time was $200 \mathrm{~min}$.

\subsubsection{Influence of simulated wastewater $\mathrm{pH}$ on total phosphorus removal rate}

The experimental conditions were fixed, that is, the concentration of phosphorus-containing simulated wastewater was $10 \mathrm{mg} \cdot \mathrm{L}^{-1}$, the volume was $50 \mathrm{~mL}$, the fixed dosage of modified eggshell powder adsorbent was $4.0 \mathrm{~g}$, the temperature of the shaker reactor was $25^{\circ} \mathrm{C}$, the speed was $160 \mathrm{r} \cdot \mathrm{min}^{-1}$, the adsorption time was 200 $\min$.

\subsubsection{Influence of the adsorption time on total phosphorus removal rate}

The experimental conditions were fixed, that is, the concentration of phosphorus-containing simulated wastewater was $10 \mathrm{mg} \cdot \mathrm{L}^{-1}, \mathrm{pH}$ value was adjusted to 2 , the volume was $50 \mathrm{~mL}$, the fixed dosage of modified eggshell powder adsorbent was $4.0 \mathrm{~g}$, the temperature of the shaker reactor was $25^{\circ} \mathrm{C}$, the speed was $160 \mathrm{r} \cdot \mathrm{min}^{-1}$.

\subsection{Orthogonal tests}

To determine the optimal conditions for total phosphorus removal, four factors and three levels orthogonal test was detailed in Table 1.

\subsection{Best Process Validation}

The experiments at the optimal conditions were repeated for 5 times. The average adsorption rate and relative standard deviation were calculated to verify the stability of the optimal process.

\subsection{Comparison of phosphorus removal performance of eggshell before and after modification}

Under the same experimental conditions, eggshell powder adsorbents before and after modification were added to compare the effects of adsorption before and after modification. 


\subsection{Sample characterization}

$\mathrm{XRD}$, FTIR and SEM were used to characterize the adsorbent before and after modification.

\section{Results and disussion}

\subsection{Characterization of adsorbents}

\subsubsection{Characterization by Fourier infrared spectroscopy (FTIR)}

Figure 1 illustrates that the types of functional groups on the surface of the eggshell remained basically the same before and after modification, but the symmetrical or asymmetrical stretching vibration absorption peaks of ionized carboxyl groups appeared after modification, which enhanced the polarity of the surface and was beneficial to the adsorption of phosphorus. The figure 1 shows that the main absorption peak at $1428 \mathrm{~cm}^{-1}$, illustrate the main existence of eggshell matrix is carbonate minerals. There were also absorption peaks at $875 \mathrm{~cm}^{-1}$ and $712 \mathrm{~cm}^{-1}$ caused by the presence of large amounts of calcium carbonate in-plane deformation and out-plane deformation mode ${ }^{[14]}$. There are symmetric or asymmetric stretching vibration absorption peaks of ionized carboxyl groups at $1638 \mathrm{~cm}^{-1}$ and $1440 \mathrm{~cm}^{-1}$. There is also an absorption peak at $3461 \mathrm{~cm}^{-1}$, which may be the absorption peak of the stretching vibration of the hydroxyl group (alcohol-phenolic and carboxylic acid), and the extension vibration absorption peak of C$\mathrm{H}$ near $2870 \mathrm{~cm}^{-1}$ and $2850 \mathrm{~cm}^{-1}$. The existence of these functional groups can effectively improve the adsorption and removal rate of phosphorus.

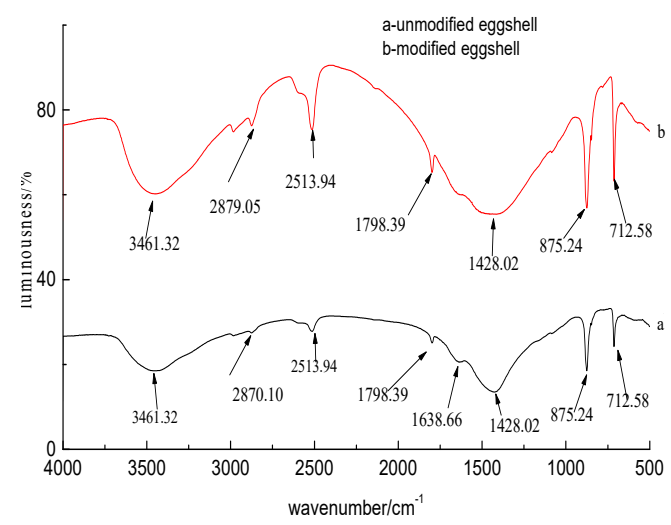

Fig. 1. Fourier infrared spectroscopy of eggshell before and after modification

\subsubsection{Characterization by $X$-ray diffraction $(X R D)$}

Figure 2 shows that when the angle of $2 \theta$ is $29.4^{\circ}, 39.5^{\circ}$, $43.2^{\circ}, 48.6^{\circ}, 60.7^{\circ}$ and $64.8^{\circ}$, the peaks are all diffraction peaks of calcium carbonate, the main component of eggshel[ ${ }^{[15]}$. The number of diffraction peaks did not change significantly before and after modification, but the eggshell adsorbent modified by potassium permanganate had a strong, sharp diffraction peak and the crystal is intact.

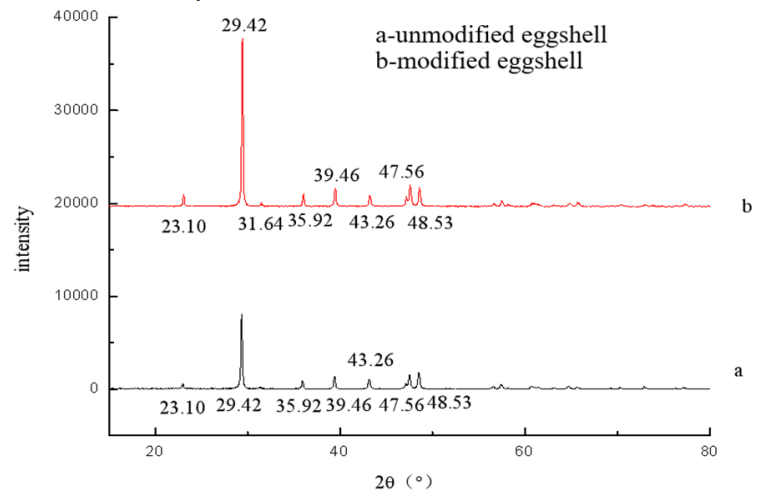

Fig. 2. X-ray diffraction pattern of eggshell before and after modification

\subsubsection{Characterization by Scanning electron microscope}

Figure 3 shows the scanning electron microscope (SEM) spectra of eggshell with a $17000 x$ magnification. As shown in spectrogram a, the surface of the original eggshell presents a whole structure, which is uniform and orderly, and there are many small holes with uniform size of micro. As shown in spectrogram $b$, there are many particle deposits on the surface of eggshell modified by potassium permanganate, which makes the structure more loose, the number of pores and the specific surface area increase. It is beneficial to increase the adsorption and removal rate of phosphorus. As shown in spectrogram c, the surface roughness of modified eggshell is reduced after phosphorus removal by adsorption, and the surface roughness becomes smoothed and the pores are obviously reduced, indicating that the adsorption effect of phosphorus removal is better.

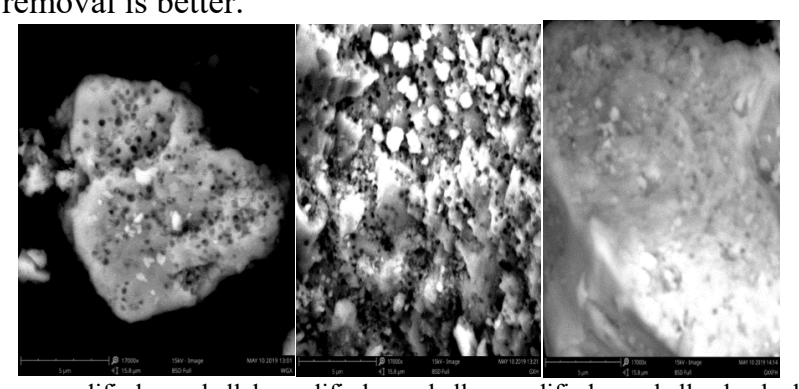

a-unmodified eggshell b-modified eggshell c-modified eggshell adsorbed by phosphorus

Fig. 3. Scanning electron microscope spectrogram of eggshell before and after adsorption 


\subsection{Study on single factor of adsorption performance}

\subsubsection{Effect of initial phosphorus concentration}

Figure 4 shows the effect of initial total phosphorus concentration on removal efficiency. With increasing concentrations, the removal efficiency of phosphorus decreases and the adsorptive capacity increases. This is because that there is a limit to the number of holes, functional groups of the modified eggshell, and with the increase of phosphorus concentration in the simulated wastewater containing phosphorus, the number of adsorbate in the solution will increase, resulting in insufficient reaction with adsorbents, so the adsorption rate decreases.

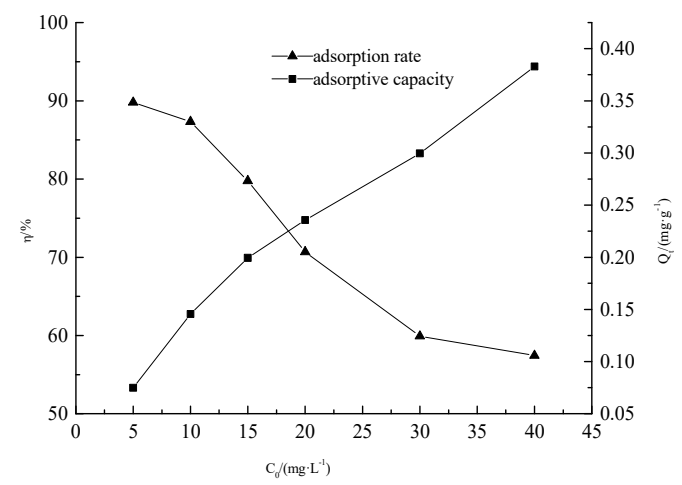

Fig. 4. Effect of initial phosphorus concentration on adsorption properties

\subsubsection{Effect of adsorbent dosage}

Figure 5 shows the effect of adsorbent dosage on total phosphorus removal efficiency. According to our observations, total phosphorus removal efficiency increased firstly and then tended to balance with the increase of the amount of adsorbent, but the adsorptive capacity showed a downward trend. This is because the number of functional groups, adsorption sites and pores will also increase accordinglywith the increase of the amount of adsorbent, so that the adsorption rate will increase. When the equilibrium state is reached, the adsorption rate will remain unchanged slowly. According to the meaning of adsorptive capacity, increase the amount of adsorbent, the amount of phosphorus adsorbed per unit mass of the adsorbent decreases.

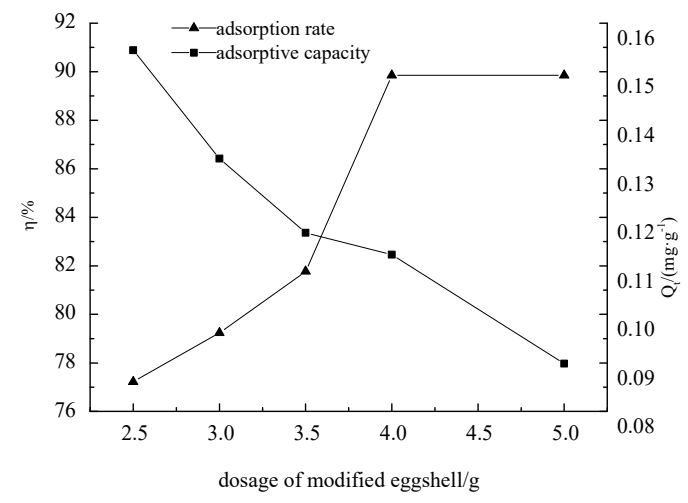

Fig. 5. Effect of adsorbent dosage on adsorption properties

\subsubsection{Effect of simulated wastewater $\mathrm{pH}$}

Figure 6 shows the effect of simulated wastewater $\mathrm{pH}$ on total phosphorus removal efficiency. The adsorption rate and amount decreased with the increase of $\mathrm{pH}$ value, because the calcium carbonate in the eggshell dissolved into calcium ions under acidic conditions, and the phosphate ion in the solution was easy to combine with calcium ions to form $\mathrm{Ca}_{3}\left(\mathrm{PO}_{4}\right)_{2}$. When $\mathrm{pH}=2$, the adsorption rate reached the maximum of $96.92 \%$. The solubility degree of calcium carbonate in the eggshell is different at different $\mathrm{pH}$ values. When the $\mathrm{pH}$ is less than 6 , the calcium carbonate in the eggshell is easy to dissolve into $\mathrm{Ca}^{2+}$, easier to adsorb phosphorus, the adsorption rate is higher, when the $\mathrm{pH}$ is greater than 6 , calcium carbonate in eggshell is difficult to dissolve, so there are less substances that can adsorb phosphorus, resulting in a decrease in adsorption rate.

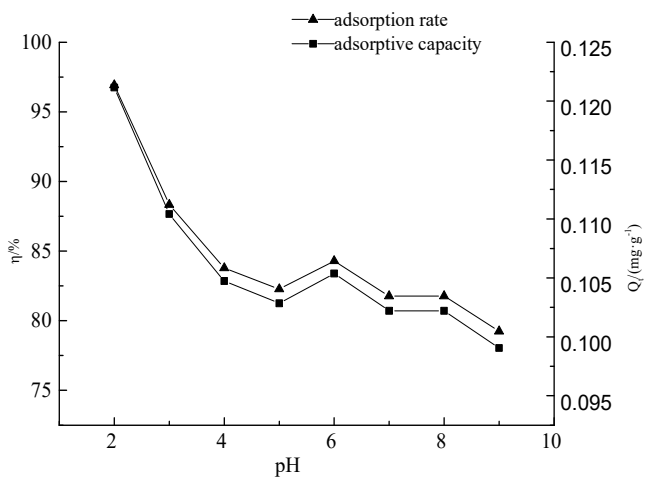

Fig. 6. Effect of $\mathrm{pH}$ on adsorption properties

\subsubsection{Effect of the adsorption time}

Figure 7 shows the effect of the adsorption time on total phosphorus removal efficiency. When the adsorption time is $450 \mathrm{~min}$, the adsorption rate and adsorptive capacity of the adsorbent are the maximum, indicating that the adsorption of phosphorus by the adsorbent has reached saturation. When the adsorption time exceeded $450 \mathrm{~min}$, the adsorption rate showed a decreasing trend, 
indicating that desorption phenomenon occurred, resulting in phosphate ions desorption from the surface of the modified eggshell powder adsorbent and returned to the solution, so both adsorption rate and adsorptive capacity decreased.

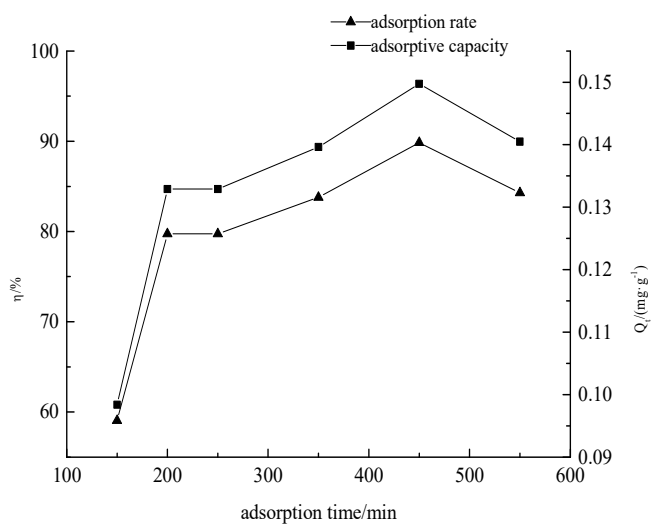

\subsection{Orthogonal tests}

The analysis of orthogonal experimental data is shown in Table 1.

Fig. 7. Effect of the adsorption time on adsorption properties

\begin{tabular}{|c|c|c|c|c|c|}
\hline No. & Adsorbent dosage(g) & $\begin{array}{l}\text { Wastewater } \\
\text { concentration } \\
\left(\mathrm{mg} \cdot \mathrm{L}^{-1}\right)\end{array}$ & $\mathrm{pH}$ & $\begin{array}{l}\text { adsorption } \\
\text { time(min) }\end{array}$ & $\begin{array}{l}\text { adsorption rate } \\
(\%)\end{array}$ \\
\hline 1 & 3.5 & 5 & 2 & 200 & 90.81 \\
\hline 2 & 3.5 & 10 & 3 & 350 & 84.29 \\
\hline 3 & 3.5 & 15 & 4 & 450 & 74.71 \\
\hline 4 & 4.0 & 5 & 3 & 450 & 83.74 \\
\hline 5 & 4.0 & 10 & 4 & 200 & 78.74 \\
\hline 6 & 4.0 & 15 & 2 & 350 & 85.15 \\
\hline 7 & 4.5 & 5 & 4 & 350 & 82.73 \\
\hline 8 & 4.5 & 10 & 2 & 450 & 89.85 \\
\hline 9 & 4.5 & 15 & 3 & 200 & 79.76 \\
\hline $\mathrm{K}_{1}$ & 249.81 & 257.27 & 265.81 & 249.31 & \\
\hline $\mathrm{K}_{2}$ & 247.63 & 252.88 & 247.79 & 252.17 & \\
\hline $\mathrm{K}_{3}$ & 252.34 & 239.63 & 236.18 & 248.30 & \\
\hline $\mathrm{k}_{1}$ & 83.27 & 85.76 & 88.60 & 83.10 & \\
\hline $\mathrm{k}_{2}$ & 82.54 & 84.29 & 82.60 & 84.06 & \\
\hline $\mathrm{k}_{3}$ & 84.11 & 79.88 & 78.73 & 82.77 & \\
\hline $\mathrm{R}$ & 1.57 & 5.88 & 9.88 & 1.29 & \\
\hline $\begin{array}{l}\text { As in } \\
\text { acess ce } \\
\text { ass of t } \\
\text { istewat }\end{array}$ & $\begin{array}{l}\text { ed by Table 1, th } \\
\text { on for phosphorus } \\
\text { odified eggshell ads } \\
\text { acentration of } 5 \mathrm{mg} \\
\text { orption time of } 350 \\
\text { results, the factor }\end{array}$ & $\begin{array}{l}\text { optimal adsorption } \\
\text { moval are as follows: } \\
\text { bent of } 4.5 \mathrm{~g} \text {, initial } \\
-1 \text {, wastewater } \mathrm{pH} \text { of } \\
\text { in. According to the } \\
\text { affecting adsorption }\end{array}$ & \multicolumn{3}{|c|}{$\begin{array}{l}\text { and phosphorus removal exhibit the following order: } \\
\text { wastewater } \mathrm{pH}>\text { wastewater concentration }>\text { adsorbent } \\
\text { dosage }>\text { adsorption time. } \\
\text { To verify the stability of the optimal adsorption } \\
\text { process, five parallel experiments were conducted, and } \\
\text { the results were shown in Table } 2 \text {. As indicated by Table } \\
2 \text {, the optimal adsorption process is rathe stable. }\end{array}$} \\
\hline
\end{tabular}


Table 2. Stability verification of optimum process conditions

\begin{tabular}{llll}
\hline No. & & $\begin{array}{l}\text { the average adsorption } \\
\text { rate }(\%)\end{array}$ & \\
\hline 1 & 90.81 & & \\
2 & 90.66 & 90.78 & 0.065 \\
3 & 90.79 & & \\
4 & 90.81 & & \\
5 & 90.81 & & \\
\hline
\end{tabular}

\subsection{Comparison of phosphorus removal effect of eggshell before and after modification}

Figure 8 shows that the adsorption effect of the modified eggshell on phosphorus was significantly enhanced, and the adsorption rate was 13 times higher than that before the modification. This is mainly due to the loose and porous surface of the modified eggshell, because the modification produces some new adsorption sites, which is conducive to the coordination reaction with phosphate in water, so as to achieve the purpose of phosphorus removal mainly through the way of chemical adsorption.

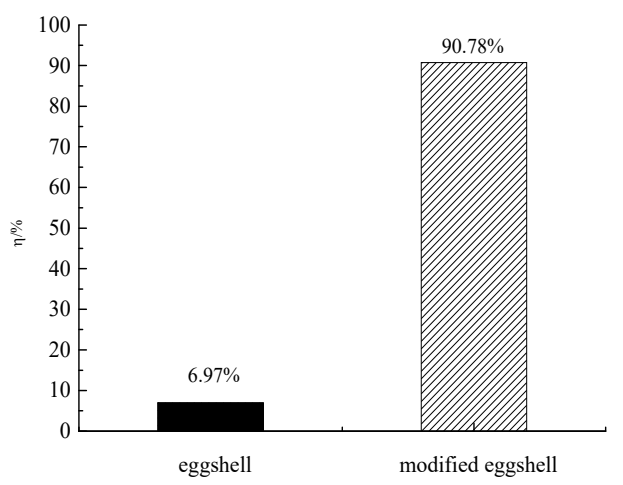

Fig. 8. Comparison of phosphorus adsorption effect before and after modification

\section{Conclusions}

The study of treating phosphorus wastewater with potassium permanganate modified eggshell as adsorbent has not been reported. Compared with unmodified eggshell, the adsorption rate increased by more than 13 times. As a kind of kitchen waste, eggshell can cause environmental pollution if it is not properly treated. The adsorbents were prepared by modified eggshell and used in the treatment of phosphorus containing wastewater, which achieved good results and realized the resource utilization of eggshell. At the same time, it provides a new way for the treatment of phosphorus containing wastewater.

Foundation item: the Research Basic Ability Enhancement Project of Young and Middle-aged Teachers in Guangxi Universities in 2021(No.2021KY0616).

\section{References}

1. J. Carvalho, J. Araujo, F. Castro. Was. Bio. V. 2 (2011).

2. D. Kim, Y. Kim, J.H. Choi, K.S. Ryoo, Bull. Korean Chem. Soc. 41(2020).

3. N.Y. Mezenner, A. Bensmaili. Chem. Eng. J. 147,2(1996).

4. M. Gibbs, D.Özkundakci. Hydro. 661,1(2011).

5. E. Kurzbaum, O. Bar Shalom. Appl. Clay Sci. 123(2016).

6. S.C. Kang, B.H. Lee. Environ. Eng. Res. 17, 4(2012).

7. W.T. Tsai, K.J. Hsien, H.C. Hsu, C.M. Lin, K.Y. Lin, C.H. Chiu. Bio. Tech. 99, 6(2008).

8. A. Animesh. Int. J. Res. Chem. Environ. 3, 1(2013).

9. M. Ahmad, A.R.A. Usman, S.S. Lee, S. Kim, J. Joo, J.E. Yang, Y.S. Ok. J. Indus. Eng. Chem. 18, 1(2012).

10. PH.J. ARK, S.W. JEONG, YJ.K. ANG, B.G. KIM, S.M. LEE. J. Environ. Sci. 19, 12(2007).

11. I.A. Oke, N.O. Olarinoye, S.R.A. Adewusi. Adsorp. 14, 1(2008).

12. N.Y. Mezenner, A. Bensmaili. Chem. Engin. J. 147, 2(2009).

13. R.A. Lelifajri. Rasayan J. Chem. 10, 2(2017).

14. W.T. Tsai, J.M. Yang, C.W. Lai, Y.H. Cheng, C.C. Lin , C.W. Yeh. Bio. Technol. 97, 3(2006).

15. J.V. F. Cano, R. L. Ramos, J. M. Barron, R.M. G. Coronado, A. A. Piña, G.J. L. Delgado. Appl. Surf. Sci. 276, 2013. 